\title{
MUTATIONS OF FAKE WEIGHTED PROJECTIVE PLANES
}

\author{
MOHAMMAD E. AKHTAR AND ALEXANDER M. KASPRZYK
}

\begin{abstract}
In previous work by Coates, Galkin, and the authors, the notion of mutation between lattice polytopes was introduced. Such a mutation gives rise to a deformation between the corresponding toric varieties. In this paper we study one-step mutations that correspond to deformations between weighted projective planes, giving a complete characterisation of such mutations in terms of $T$-singularities. We show also that the weights involved satisfy Diophantine equations, generalising results of Hacking-Prokhorov.
\end{abstract}

\section{INTRODUCTION}

In [ACGK12] we described a combinatorial notion of mutation between convex lattice polytopes. In this paper we begin to explore the geometry behind this idea. Given a convex lattice polytope $P$ containing the origin and with primitive vertices, there is a corresponding toric variety $X$ defined by the spanning fan of $P$. A mutation between polytopes $P$ and $Q$ determines a deformation between $X_{P}$ and $X_{Q}$ [It12]. Our main result characterises mutations between triangles; thus we characterise certain deformations, over $\mathbb{P}^{1}$, with fibers given by fake weighted projective planes. We recover and generalise certain results of Hacking and Prokhorov [HP10, Theorem 4.1] connecting the fake weighted projective planes with $T$-singularities to solutions of Markov-type equations. We prove the following:

Proposition 1.1. Let $X=\mathbb{P}\left(\lambda_{0}, \lambda_{1}, \lambda_{2}\right)$ be a weighted projective plane. Up to reordering of the weights, there exists a one-step mutation to a weighted projective plane $Y$ if and only if $\frac{1}{\lambda_{0}}\left(\lambda_{1}, \lambda_{2}\right)$ is a T-singularity. When this is the case, $Y=\mathbb{P}\left(\lambda_{1}, \lambda_{2}, \frac{\left(\lambda_{1}+\lambda_{2}\right)^{2}}{\lambda_{0}}\right)$. More generally, there exists a one-step mutation from the fake weighted projective plane $X /(\mathbb{Z} / n)$ to the fake weighted projective plane $Y /\left(\mathbb{Z} / n^{\prime}\right)$ only if $n=n^{\prime}$ and $\frac{1}{\lambda_{0}}\left(\lambda_{1}, \lambda_{2}\right)$ is a T-singularity.

In Proposition 3.12 we associate to a weighted projective plane $X$ a Diophantine equation

$$
m x_{0} x_{1} x_{2}=k\left(c_{0} x_{0}^{2}+c_{1} x_{1}^{2}+c_{2} x_{2}^{2}\right) .
$$

The weights $\left(\lambda_{0}, \lambda_{1}, \lambda_{2}\right)$ of $X$ correspond to a solution $\left(a_{0}, a_{1}, a_{2}\right)$, where $\lambda_{i}=c_{i} a_{i}^{2}, i=0,1,2$, and the degree of $X$ is given by

$$
\left(-K_{X}\right)^{2}=\frac{m^{2}}{c_{0} c_{1} c_{2} k^{2}} .
$$

One-step mutations of $X$ correspond to transformations of the solutions to (1.1), and all such solutions can be generated from the so-called minimal weights by mutation. 
When $X=\mathbb{P}^{2}$, equation (1.1) becomes the celebrated Markov equation Mar80. Certain other special cases were studied by Rosenberger [Ros79]. These cases all have finitely many minimal weights. In 4 we give an example where the corresponding Diophantine equation has infinitely many minimal weights.

\section{Mutations of Fano polytopes}

Let $N \cong \mathbb{Z}^{n}$ be a lattice with dual $M:=\operatorname{Hom}(N, \mathbb{Z})$. A lattice polytope $P \subset N_{\mathbb{Q}}:=N \otimes_{\mathbb{Z}} \mathbb{Q}$ is called Fano if it satisfies three conditions:

(1) $P$ is of maximum dimension, $\operatorname{dim} P=\operatorname{dim} N$;

(2) The origin is contained in the strict interior of $P, \mathbf{0} \in \operatorname{int}(P)$;

(3) The vertices vert $(P)$ of $P$ are primitive lattice points, i.e. for any $v \in \operatorname{vert}(P)$ there are no other lattice points on the line segment $\overline{\mathbf{0} v}$ joining $v$ and the origin.

The dual of $P$ is defined to be the polyhedron

$$
P^{\vee}:=\left\{u \in M_{\mathbb{Q}} \mid u(v) \geqslant-1 \text { for all } v \in P\right\} \subset M_{\mathbb{Q}} .
$$

By condition (2) this is a polytope with $\mathbf{0} \in \operatorname{int}\left(P^{\vee}\right)$, although it need not be a lattice polytope. See [KN12] for an overview of Fano polytopes.

We briefly recall the notation of [ACGK12, §3]. Any choice of primitive vector $w \in M$ determines a lattice height function $w: N \rightarrow \mathbb{Z}$ which naturally extends to $N_{\mathbb{Q}} \rightarrow \mathbb{Q}$. A subset $S \subset N_{\mathbb{Q}}$ is said to lie at height $h \in \mathbb{Q}$ with respect to $w$ if $w(S):=\{w(s) \mid s \in S\}=\{h\}$; we write $w(S)=h$. The set of all points of $N_{\mathbb{Q}}$ lying at height $h$ with respect to a given $w$ is an affine hyperplane $H_{w, h}:=\left\{v \in N_{\mathbb{Q}} \mid w(v)=h\right\}$. In particular,

$$
w_{h}(P):=\operatorname{conv}\left(H_{w, h} \cap P \cap N\right) \subset N_{\mathbb{Q}}
$$

will denote the (possibly empty) convex hull of all lattice points in $P$ at height $h$.

Define

$$
h_{\min }:=\min \{w(v) \mid v \in P\}, \quad h_{\max }:=\max \{w(v) \mid v \in P\} .
$$

Since $P$ is a lattice polytope, both $h_{\min }$ and $h_{\max }$ are integers. Condition (2) guarantees that $h_{\min }<0$ and $h_{\max }>0$.

Definition 2.1. A factor of $P$ with respect to $w$ is a lattice polytope $F \subset N_{\mathbb{Q}}$ satisfying:

(1) $w(F)=0$;

(2) For every integer $h, h_{\min } \leqslant h<0$, there exists a (possibly empty) lattice polytope $G_{h} \subset N_{\mathbb{Q}}$ at height $h$ such that

$$
H_{w, h} \cap \operatorname{vert}(P) \subseteq G_{h}+(-h) F \subseteq w_{h}(P) .
$$

Note that, for given polytope $P \subset N_{\mathbb{Q}}$ and width vector $w \in M$, a factor $F$ need not exist. When a factor does exist we make the following construction: 
Definition 2.2 ([ACGK12, Definition 5]). Let $P \subset N_{\mathbb{Q}}$ be a polytope with width vector $w \in M$, factor $F$, and polytopes $\left\{G_{h}\right\}$. We define the corresponding combinatorial mutation to be the convex lattice polytope

$$
\operatorname{mut}_{w}\left(P, F ;\left\{G_{h}\right\}\right):=\operatorname{conv}\left(\bigcup_{h=h_{\min }}^{-1} G_{h} \cup \bigcup_{h=0}^{h_{\max }}\left(w_{h}(P)+h F\right)\right) \subset N_{\mathbb{Q}} .
$$

For brevity we will refer to a combinatorial mutation simply as a mutation.

We summarise the key properties of mutation ACGK12:

(1) Since for any $v \in N$ such that $w(v)=0$ we have that

$$
\operatorname{mut}_{w}\left(P, F ;\left\{G_{h}\right\}\right) \cong \operatorname{mut}_{w}\left(P, v+F ;\left\{G_{h}+h v\right\}\right),
$$

we need only consider factors $F$ up to translation. In particular, choosing $F$ to be a point leaves $P$ unchanged (up to isomorphism).

(2) If $\left\{G_{h}\right\}$ and $\left\{G_{h}^{\prime}\right\}$ are any two collections of polytopes for a factor $F$, then

$$
\operatorname{mut}_{w}\left(P, F ;\left\{G_{h}\right\}\right) \cong \operatorname{mut}_{w}\left(P, F ;\left\{G_{h}^{\prime}\right\}\right) .
$$

Thus the choice of collection $\left\{G_{h}\right\}$ is irrelevant and we $\operatorname{write} \operatorname{mut}_{w}(P, F)$.

(3) $P$ is a Fano polytope if and only if $\operatorname{mut}_{w}(P, F)$ is a Fano polytope.

(4) Let $Q:=\operatorname{mut}_{w}(P, F)$. Then $\operatorname{mut}_{-w}(Q, F)=P$, so mutations are invertible.

In [ACGK12] it was also shown that mutations have a natural description as a piecewise linear transformation of the lattice $M$. We require the following definition.

Definition 2.3. The inner normal fan in $M$ of a polytope $F \subset N_{\mathbb{Q}}$ is generated by the cones $\sigma_{v_{F}}$ consisting of those linear functions which are minimal on a given vertex $v_{F}$ of $F$. That is,

$$
\sigma_{v_{F}}:=\left\{u \in M_{\mathbb{Q}} \mid u\left(v_{F}\right)=\min \left\{u\left(v^{\prime}\right) \mid v^{\prime} \in F\right\}\right\} .
$$

(5) A mutation of $P \subset N_{\mathbb{Q}}$ induces a piecewise linear transformation $\varphi$ of $M_{\mathbb{Q}}$ such that $\left(\varphi\left(P^{\vee}\right)\right)^{\vee}=\operatorname{mut}_{w}(P, F)$, given by

$$
\varphi: u \mapsto u-u_{\min } w, \quad u \in M_{\mathbb{Q}},
$$

where $u_{\min }:=\min \left\{u\left(v_{F}\right) \mid v_{F} \in \operatorname{vert}(F)\right\}$. The inner normal fan of $F \subset N_{\mathbb{Q}}$ determines a chamber decomposition of $M_{\mathbb{Q}}$, and $\varphi$ acts as a linear transformation on the interior of each maximal dimensional cone of this fan.

(6) As a consequence of (5), the toric varieties $X_{P}$ and $X_{Q}$ defined by the spanning fans of $P$ and $Q:=\operatorname{mut}_{w}(P, F)$ have the same degree (in fact they have the same Hilbert series).

Example 2.4. Consider the triangle $P=\operatorname{conv}\{(1,-1),(-1,2),(0,-1)\} \subset N_{\mathbb{Q}}$ corresponding to the toric variety $\mathbb{P}^{2}$. Let $w=(0,1) \in M$ and set $F=\operatorname{conv}\{\mathbf{0},(1,0)\} \subset N_{\mathbb{Q}}$. This defines a mutation from $P$ to the triangle $Q=\operatorname{conv}\{(1,2),(-1,2),(0,-1)\} \subset N_{\mathbb{Q}}$, as illustrated in 


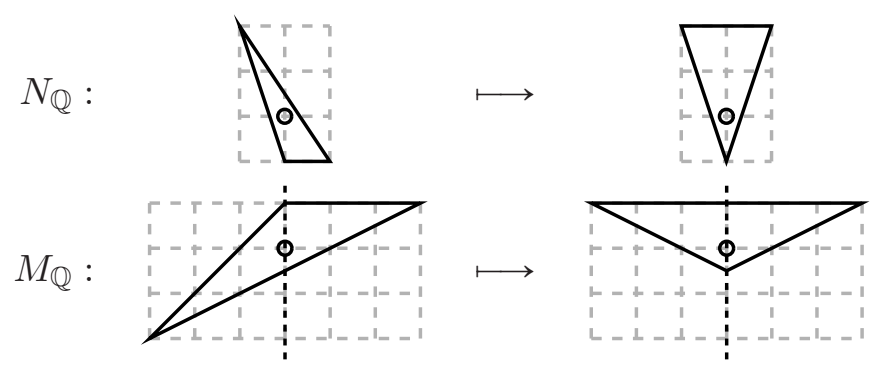

Figure 1. A mutation from the triangle associated with $\mathbb{P}^{2}$ to the triangle associated with $\mathbb{P}(1,1,4)$.

Figure 1. On the dual side, this corresponds to a piecewise linear map $\varphi: u \mapsto u M_{\sigma}$ for $u=(\alpha, \beta) \in M_{\mathbb{Q}}$, where

$$
M_{\sigma}= \begin{cases}\left(\begin{array}{ll}
1 & 0 \\
0 & 1
\end{array}\right) & \text { if } \alpha \geqslant 0 \\
\left(\begin{array}{cc}
1 & -1 \\
0 & 1
\end{array}\right) & \text { otherwise }\end{cases}
$$

In particular, $\varphi\left(P^{\vee}\right)=Q^{\vee}$.

Mutations are particularly simple in the two-dimensional case. In this setting, $w \in M$ defines a non-trivial mutation of $P \subset N_{\mathbb{Q}}$ if and only if $w \in\left\{\bar{u} \mid u \in \operatorname{vert}\left(P^{\vee}\right)\right\} \subset M$, where $\bar{u} \in M$ is the unique primitive lattice vector on the ray passing through $u$. Nontrivial factors $F \subset N_{\mathbb{Q}}$ are just line segments, so it suffices to restrict attention to those $F$ which have vertex set $\{\mathbf{0}, f\}$, for some $f \in N$ with $w(f)=0$. The inner normal fan of any factor $F$ of $P$ with respect to a given $w$ is just the linear subspace of $M_{\mathbb{Q}}$ spanned by $w$. This divides $M_{\mathbb{Q}}$ into two chambers; the piecewise linear transformation $\varphi$ acts trivially in one of the chambers, and as $u \mapsto u-u(f) w$ in the other.

\section{ONE-STEP MUTATIONS OF TRIANGLES}

Set $N \cong \mathbb{Z}^{2}$ and let $P:=\operatorname{conv}\left\{v_{0}, v_{1}, v_{2}\right\} \subset N_{\mathbb{Q}}$ be a Fano triangle. Since $\mathbf{0} \in \operatorname{int}(P)$ there exists a (unique) choice of coprime positive integers $\lambda_{0}, \lambda_{1}, \lambda_{2} \in \mathbb{Z}_{>0}$ with $\lambda_{0} v_{0}+\lambda_{1} v_{1}+\lambda_{2} v_{2}=\mathbf{0}$. The projective toric surface $X$ given by the spanning fan of $P$ has Picard rank 1 , and is called a fake weighted projective plane with weights $\left(\lambda_{0}, \lambda_{1}, \lambda_{2}\right) ; X$ is the quotient of $\mathbb{P}\left(\lambda_{0}, \lambda_{1}, \lambda_{2}\right)$ by the action of a finite group of order mult $(X)$ acting freely in codimension one Con02, Buc08, Kas09].

Remark 3.1. Since the vertices of $P$ are primitive, the weights $\left(\lambda_{0}, \lambda_{1}, \lambda_{2}\right)$ are well-formed: that is, $\operatorname{gcd}\left\{\lambda_{i}, \lambda_{j}\right\}=1, i \neq j$. In this paper we will always require that weights are well-formed.

Definition 3.2. We say that a fake weighted projective plane $Y$ with defining Fano triangle $Q \subset N_{\mathbb{Q}}$ is obtained from $X$ by a one-step mutation if $Q \cong \operatorname{mut}_{w}(P, F)$ for some choice of $w$ and factor $F$. 


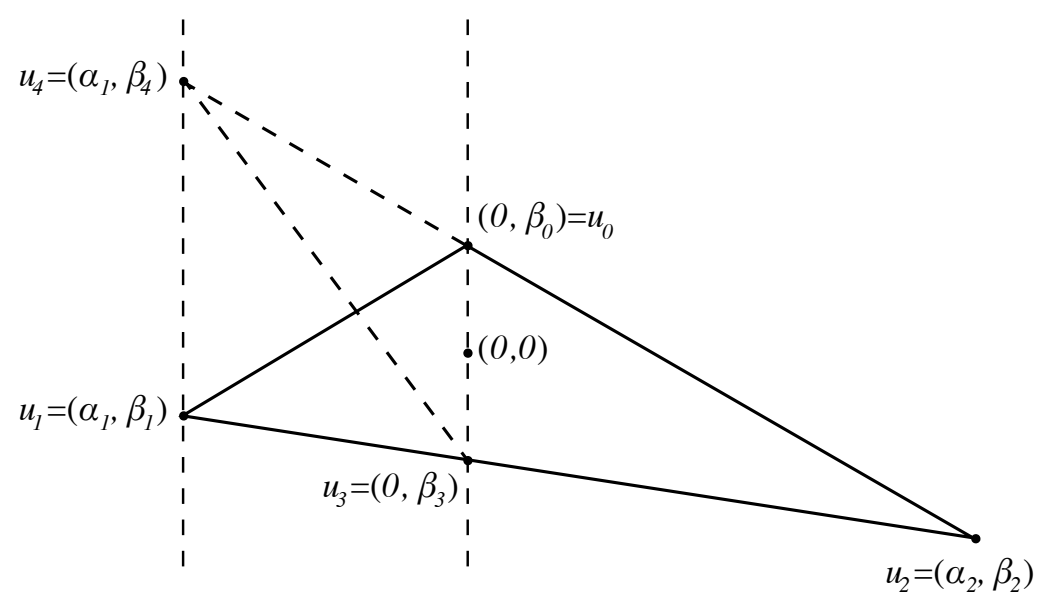

Figure 2. A one-step mutation, depicted in $M_{\mathbb{Q}}$, of the triangle $\operatorname{conv}\left\{u_{0}, u_{1}, u_{2}\right\}$ to the triangle conv $\left\{u_{2}, u_{3}, u_{4}\right\}$.

3.1. One-step mutations in $M_{\mathbb{Q}}$ and weights. First we address how the weights $\left(\lambda_{0}, \lambda_{1}, \lambda_{2}\right)$ associated with a Fano triangle $T \subset N_{\mathbb{Q}}$ transform under mutation. We will require the following fact (see, for example, Con02, Lemma 5.3]): Let $T^{\vee}=\operatorname{conv}\left\{u_{0}, u_{1}, u_{2}\right\}$ by the triangle in $M_{\mathbb{Q}}$ dual to $T$. Then, after possible reordering, $\lambda_{0} u_{0}+\lambda_{1} u_{1}+\lambda_{2} u_{2}=\mathbf{0}$. Hence the weights of $T$ and the weights of $T^{\vee}$ are equivalent.

Proposition 3.3. Let $X$ be a fake weighted projective plane with weights $\left(\lambda_{0}, \lambda_{1}, \lambda_{2}\right)$. Suppose there exists a one-step mutation to a fake weighted projective plane $Y$. Then, up to relabelling, $\lambda_{0} \mid\left(\lambda_{1}+\lambda_{2}\right)^{2}$ and $Y$ has weights

$$
\left(\lambda_{1}, \lambda_{2}, \frac{\left(\lambda_{1}+\lambda_{2}\right)^{2}}{\lambda_{0}}\right)
$$

Proof. Consider a lattice triangle $T_{1} \subset N_{\mathbb{Q}}, \mathbf{0} \in \operatorname{int}\left(T_{1}\right)$, and suppose that there exists a width vector $w \in M$ and factor $F \subset N_{\mathbb{Q}}, w(F)=0$, such that the mutation $T_{2}=\operatorname{mut}_{w}\left(T_{1}, F\right)$ is also a triangle. Without loss of generality we can assume that $w=(0,1) \in M$ and $F=\operatorname{conv}\{\mathbf{0},(a, 0)\}$ for some $a \in \mathbb{Z}_{>0}$. The mutation corresponds to a piecewise linear action on $M_{\mathbb{Q}}$ via $u \mapsto u M_{\sigma}$ given by

$$
M_{\sigma}= \begin{cases}\left(\begin{array}{cc}
1 & 0 \\
0 & 1
\end{array}\right) & \text { if } u \in M^{+} \\
\left(\begin{array}{cc}
1 & -a \\
0 & 1
\end{array}\right) & \text { otherwise }\end{cases}
$$

where $M^{+}$is the half-space $\left\{(\alpha, \beta) \in M_{\mathbb{Q}} \mid \alpha>0\right\}$. Let $T_{1}^{\vee}=\operatorname{conv}\left\{u_{0}, u_{1}, u_{2}\right\} \subset M_{\mathbb{Q}}$ be the (possibly rational) triangle dual to $T_{1}$, where $u_{2} \in M^{+}$and so is fixed under the action of the mutation, and $u_{1} \in M^{-}:=\left\{(\alpha, \beta) \in M_{\mathbb{Q}} \mid \alpha<0\right\}$. Since $T_{2}^{\vee} \subset M_{\mathbb{Q}}$ is also a triangle, the only possibility is that $u_{0}$ lies on the line $\langle w\rangle:=\left\{\gamma w \in M_{\mathbb{Q}} \mid \gamma \in \mathbb{Q}\right\}, T_{2}^{\vee}=\operatorname{conv}\left\{u_{2}, u_{3}, u_{4}\right\}$ where $u_{0}$ is contained in the line segment $\overline{u_{2} u_{4}}$ joining $u_{2}$ and $u_{4}$, and $u_{3}$ is contained in the line segment $\overline{u_{1} u_{2}}$. This situation is illustrated in Figure 2. 
Since $\mathbf{0} \in T_{1}^{\vee}$ there exist unique weights $\left(\lambda_{0}, \lambda_{1}, \lambda_{2}\right) \in \mathbb{Z}_{>0}^{3}, \operatorname{gcd}\left\{\lambda_{0}, \lambda_{1}, \lambda_{2}\right\}=1$, such that

$$
\lambda_{0} u_{0}+\lambda_{1} u_{1}+\lambda_{2} u_{2}=\mathbf{0} .
$$

Since $u_{3}=\left(0, \beta_{3}\right) \in \overline{u_{1} u_{2}}$ there exists some $0<\mu<1$ such that $\mu \alpha_{1}+(1-\mu) \alpha_{2}=0$. But $\lambda_{1} \alpha_{1}+\lambda_{2} \alpha_{2}=0$, hence

By uniqueness of $\mu$,

$$
\frac{\lambda_{1}}{\lambda_{1}+\lambda_{2}} \alpha_{1}+\frac{\lambda_{2}}{\lambda_{1}+\lambda_{2}} \alpha_{2}=0
$$

$$
u_{3}=\frac{\lambda_{1}}{\lambda_{1}+\lambda_{2}} u_{1}+\frac{\lambda_{2}}{\lambda_{1}+\lambda_{2}} u_{2}
$$

Similarly, since $u_{0}=\left(0, \beta_{0}\right) \in \overline{u_{2} u_{4}}$ there exists some $0<\nu<1$ such that $u_{0}=\nu u_{2}+(1-\nu) u_{4}$, giving

Comparing coefficients we see that

$$
u_{4}=\frac{1}{1-\nu} u_{0}-\frac{\nu}{1-\nu} u_{2}
$$

$$
\alpha_{1}=-\frac{\nu}{1-\nu} \alpha_{2}
$$

But $u_{4}=u_{1}+\kappa u_{0}$ for some $\kappa>0$. Combining this with equation (3.1) we see that

$$
u_{4}=\frac{\lambda_{1} \kappa-\lambda_{0}}{\lambda_{1}} u_{0}-\frac{\lambda_{2}}{\lambda_{1}} u_{2}
$$

Comparing coefficients, we obtain

$$
\alpha_{1}=-\frac{\lambda_{2}}{\lambda_{1}} \alpha_{2}
$$

Equating equations (3.3) and (3.4) gives

$$
u_{4}=\frac{\lambda_{1}+\lambda_{2}}{\lambda_{1}} u_{0}-\frac{\lambda_{2}}{\lambda_{1}} u_{2} .
$$

Notice that, since both $u_{0}$ and $u_{3}$ are contained in $\langle w\rangle$, there exists some $\gamma>0$ such that $-\gamma u_{3}=u_{0}$. Substituting into equation (3.5) we have

$$
\frac{\lambda_{2}}{\lambda_{1}} u_{2}+u_{4}+\gamma^{\prime} u_{3}=\mathbf{0}
$$

where $\gamma^{\prime}=\gamma\left(\lambda_{1}+\lambda_{2}\right) / \lambda_{1}>0$. Substituting in equation (3.2) we obtain

$$
\frac{\lambda_{2}}{\lambda_{1}} u_{2}+u_{4}+\frac{\gamma^{\prime} \lambda_{1}}{\lambda_{1}+\lambda_{2}} u_{1}+\frac{\gamma^{\prime} \lambda_{2}}{\lambda_{1}+\lambda_{2}} u_{2}=\mathbf{0}
$$

Using equation (3.5) to rewrite the first two terms and clearing denominators gives:

$$
\left(\lambda_{1}+\lambda_{2}\right)^{2} u_{0}+\gamma^{\prime} \lambda_{1}^{2} u_{1}+\gamma^{\prime} \lambda_{1} \lambda_{2} u_{2}=\mathbf{0} .
$$

Set $h:=\lambda_{0}+\lambda_{1}+\lambda_{2}$ and $\Gamma:=\left(\lambda_{1}+\lambda_{2}\right)^{2}+\gamma^{\prime} \lambda_{1}^{2}+\gamma^{\prime} \lambda_{1} \lambda_{2}$. By comparing equations (3.1) and (3.7), uniqueness of barycentric coordinates gives:

$$
\begin{aligned}
h\left(\lambda_{1}+\lambda_{2}\right)^{2} & =\Gamma \lambda_{0}, \\
h \gamma^{\prime} \lambda_{1}^{2} & =\Gamma \lambda_{1}, \\
h \gamma^{\prime} \lambda_{1} \lambda_{2} & =\Gamma \lambda_{2} .
\end{aligned}
$$


In particular,

$$
\gamma^{\prime}=\frac{\left(\lambda_{1}+\lambda_{2}\right)^{2}}{\lambda_{0} \lambda_{1}}
$$

Substituting this expression for $\gamma^{\prime}$ back into equation (3.6) gives

$$
\lambda_{0} \lambda_{2} u_{2}+\left(\lambda_{1}+\lambda_{2}\right)^{2} u_{3}+\lambda_{0} \lambda_{1} u_{4}=\mathbf{0} .
$$

Finally, we consider the situation where $T_{1} \subset N_{\mathbb{Q}}$ is the triangle associated with a fake weighted projective plane with weights $\left(\lambda_{0}, \lambda_{1}, \lambda_{2}\right)$, and assume that there exists a one-step mutation to some triangle $T_{2} \subset N_{\mathbb{Q}}$. If $\lambda_{0}$ does not divide $\left(\lambda_{1}+\lambda_{2}\right)^{2}$, then by equation (3.8) the associated weights are

$$
\left(\lambda_{0} \lambda_{1}, \lambda_{0} \lambda_{2},\left(\lambda_{1}+\lambda_{2}\right)^{2}\right) \text {, }
$$

and these fail to be well-formed when $\lambda_{0}>1$. Therefore, we must have $\lambda_{0} \mid\left(\lambda_{1}+\lambda_{2}\right)^{2}$, giving weights

$$
\left(\lambda_{1}, \lambda_{2}, \frac{\left(\lambda_{1}+\lambda_{2}\right)^{2}}{\lambda_{0}}\right)
$$

Remark 3.4. Let $\left(\lambda_{0}, \lambda_{1}, \lambda_{2}\right)$ be well-formed weights such that $\lambda_{0} \mid\left(\lambda_{1}+\lambda_{2}\right)^{2}$, and suppose that there exists some prime $p$ such that

$$
p \mid \lambda_{1} \quad \text { and } \quad p \mid \frac{\left(\lambda_{1}+\lambda_{2}\right)^{2}}{\lambda_{0}} .
$$

Then $p \mid \lambda_{2}^{2}$ and so $p \mid \lambda_{2}$. But this contradicts $\left(\lambda_{0}, \lambda_{1}, \lambda_{2}\right)$ being well-formed. Hence

$$
\left(\lambda_{1}, \lambda_{2}, \frac{\left(\lambda_{1}+\lambda_{2}\right)^{2}}{\lambda_{0}}\right)
$$

are also well-formed.

Example 3.5. There exists no one-step mutation from $\mathbb{P}(3,5,11)$ to any other weighted projective space, since $3 \nmid(5+11)^{2}, 5 \nmid(3+11)^{2}$, and $11 \nmid(3+5)^{2}$.

Example 3.6. The requirement that $\lambda_{0} \mid\left(\lambda_{1}+\lambda_{2}\right)^{2}$ in Proposition 3.3 is necessary but not sufficient. For example, consider the triangle $T=\operatorname{conv}\{(10,-7),(-5,2),(0,1)\} \subset N_{\mathbb{Q}}$. This has weights $(1,2,3)$, however there exist no one-step mutations from $T$.

3.2. One-step mutations in $N_{\mathbb{Q}}$ and $T$-singularities. Our aim in this section is to characterise when a mutation exists. In order to do this, we require the definition of a $T$-singularity.

Definition 3.7 ([KSB88, Definition 3.7]). A quotient surface singularity is called a T-singularity if it admits a $\mathbb{Q}$-Gorenstein one-parameter smoothing.

$T$-singularities include the du Val singularities $\frac{1}{r}(1, r-1)$, and are cyclic quotient singularities of the form $\frac{1}{n d^{2}}(1, d n a-1)$, where $\operatorname{gcd}\{d, a\}=1$ [KSB88, Proposition 3.10].

Lemma 3.8. An isolated quotient singularity $\frac{1}{r}(a, b)$ is a T-singularity if and only if $r \mid(a+b)^{2}$. 
Proof. We begin by noting that the condition that $r \mid(a+b)^{2}$ is independent of the choice of representation of $\frac{1}{r}(a, b)$. For let $c$ be any integer coprime to $r$. Then $r \mid(a+b)^{2}$ if and only if $r \mid c^{2}(a+b)^{2}=(c a+c b)^{2}$.

Suppose we are given a $T$-singularity. Writing the singularity in the form $\frac{1}{n d^{2}}(1, d n a-1)$ where $\operatorname{gcd}\{d, a\}=1$, we see that $n d^{2} \mid d^{2} n^{2} a^{2}$. Conversely consider the isolated quotient singularity $\frac{1}{r}(a, b)$. Since $a$ is invertible $\bmod r$, we can write this as $\frac{1}{r}\left(1, b^{\prime}-1\right)$, where $b^{\prime} \equiv b a^{-1}+1(\bmod r)$. Write $r=n d^{2}$ where $n$ is square-free. Since $n d^{2} \mid b^{\prime 2}$ by assumption, we see that $n d \mid b^{\prime}$. In particular, we can express our singularity in the form $\frac{1}{n d^{2}}(1, d n \alpha-1)$ for some $\alpha \in \mathbb{Z}_{>0}$. Finally, we note that this really is a $T$-singularity: if $\operatorname{gcd}\{d, \alpha\}=c$ then we can absorb this factor into $n^{\prime}=n c^{2}$ whilst rescaling $d^{\prime}=d / c$ and $\alpha^{\prime}=\alpha / c$.

Proposition 3.9. Let $X$ be a fake weighted projective plane corresponding to a triangle $T \subset N_{\mathbb{Q}}$, and suppose that the cone $C$ spanned by an edge $E$ of $T$ corresponds to a $\frac{1}{r}(a, b)$ singularity. There exists a one-step mutation to a fake weighted projective plane $Y$ given by $\operatorname{mut}_{w}(T, F)$ with $w(E)=h_{\min }$ if and only if $\frac{1}{r}(a, b)$ is a T-singularity.

Proof. Let $X$ correspond to the lattice triangle $T=\operatorname{conv}\left\{v_{1}, v_{2}, v_{3}\right\} \subset N_{\mathbb{Q}}$, where $\mathbf{0} \in \operatorname{int}(T)$ and the vertices vert $(T) \subset N$ are all primitive. Consider the cone $C=\operatorname{cone}\left\{v_{1}, v_{2}\right\}$ spanned by the edge $E=\overline{v_{1} v_{2}}$; this is an isolated quotient singularity (possibly smooth), so is of the form $\frac{1}{r}(a, b)$ for some $r, a, b \in \mathbb{Z}_{>0}, \operatorname{gcd}\{r, a\}=\operatorname{gcd}\{r, b\}=1$.

Let $w \in M$ be a primitive lattice point such that $w\left(v_{1}\right)=w\left(v_{2}\right)=h$ for some $h<0$. Then, up to translation, there exists a factor $F \subset N_{\mathbb{Q}}, w(F)=0$, such that $T^{\prime}:=\operatorname{mut}_{w}(T, F)$ is a triangle if and only if $v_{1}+(-h) F=E$. Equivalently, if and only if $h|| E \cap N \mid-1$.

Finally, we express the values of $h$ and $|E \cap N|-1$ in terms of the singularity $\frac{1}{r}(a, b)$. Set $k:=\operatorname{gcd}\{r, a+b\}$. Then the height $h=-r / k$, and the number of points on the edge $E$ is given by

$$
\mid\{m \mid m \in\{0, \ldots, r\} \text { and }(a+b) m \equiv 0(\bmod r)\} \mid=1+\frac{r}{h}=1+k .
$$

Hence $h|| E \cap N \mid-1$ if and only if $r / k \mid k$. But $r / k \mid k$ if and only if $r \mid \operatorname{gcd}\{r, a+b\}^{2}=$ $\operatorname{gcd}\left\{r^{2},(a+b)^{2}\right\}$, and $r \mid \operatorname{gcd}\left\{r^{2},(a+b)^{2}\right\}$ if and only if $r \mid(a+b)^{2}$. The result follows by Lemma 3.8 ,

Example 3.10. Returning to Example 3.6, we see that the corresponding fake weighted projective space $X$ is a quotient of $\mathbb{P}(1,2,3)$ with mult $(X)=5$. The three singularities are $\frac{1}{5}(1,3)$, $\frac{1}{10}(1,3)$, and $\frac{1}{15}(1,11)$, none of which is a $T$-singularity.

When $X$ is a weighted projective plane, Proposition 3.9 tells us that the condition that $\lambda_{0} \mid\left(\lambda_{1}+\lambda_{2}\right)^{2}$ in Proposition 3.3 is both necessary and sufficient.

3.3. One-step mutations and Diophantine equations. Given the results of 3.1 and 33.2 , we are now in a position to relate one-step mutations of Fano triangles to solutions of certain Diophantine equations.

Lemma 3.11. Let $\left(\lambda_{0}, \lambda_{1}, \lambda_{2}\right) \in \mathbb{Z}_{>0}^{3}$ with $d=\operatorname{gcd}\left\{\lambda_{0}, \lambda_{1}, \lambda_{2}\right\}$. Write: 
(1) $\lambda_{i}=d c_{i} a_{i}^{2}$, where $a_{i}, c_{i} \in \mathbb{Z}_{>0}$ and $c_{i}$ is square-free;

(2) $\left(\lambda_{0}+\lambda_{1}+\lambda_{2}\right)^{2} /\left(\lambda_{0} \lambda_{1} \lambda_{2}\right)=m^{2} /\left(r k^{2}\right)$, where $m, k, r \in \mathbb{Z}_{>0}$ and $r$ is square-free;

(3) $c_{0} c_{1} c_{2}=g S^{2}$ and $d r=h T^{2}$, where $g, h, S, T \in \mathbb{Z}_{>0}$ and both $g$ and $h$ are square-free.

Then $\left(d a_{0}, d a_{1}, d a_{2}\right)$ is a solution to the Diophantine equation

$$
S m x_{0} x_{1} x_{2}=T k\left(c_{0} x_{0}^{2}+c_{1} x_{1}^{2}+c_{2} x_{2}^{2}\right) .
$$

Proof. By substituting expressions (11) and (3) into (2) we obtain

$$
g S^{2} m^{2}\left(d a_{0}\right)^{2}\left(d a_{1}\right)^{2}\left(d a_{2}\right)^{2}=h T^{2} k^{2}\left(c_{0}\left(d a_{0}\right)^{2}+c_{1}\left(d a_{1}\right)^{2}+c_{2}\left(d a_{2}\right)^{2}\right)^{2} .
$$

Comparing square-free parts, we conclude that $g=h$. Cancelling and taking square-roots on both sides establishes the result.

Since the weights are assumed to be well-formed, $d=S=T=1$ and equation (3.9) becomes

$$
m x_{0} x_{1} x_{2}=k\left(c_{0} x_{0}^{2}+c_{1} x_{1}^{2}+c_{2} x_{2}^{2}\right) .
$$

Suppose that $\left(a_{0}, a_{1}, a_{2}\right)$ is a positive integral solution to equation (3.10), so that $\lambda_{i}=c_{i} a_{i}^{2}$. The expression

$$
\frac{\left(\lambda_{0}+\lambda_{1}+\lambda_{2}\right)^{2}}{\lambda_{0} \lambda_{1} \lambda_{2}}
$$

occurring in Lemma 3.11 is equal to the degree of $\mathbb{P}\left(\lambda_{0}, \lambda_{1}, \lambda_{2}\right)$. More generally if $X$ is a fake weighted projective plane with weights $\left(\lambda_{0}, \lambda_{1}, \lambda_{2}\right)$ then (3.11) is equal to $\operatorname{mult}(X)\left(-K_{X}\right)^{2}$.

Proposition 3.12. Let $X$ be a fake weighted projective plane and suppose that there exists a one-step mutation to a fake weighted projective plane $Y$. Then the weights of $X$ and $Y$ give solutions to the same Diophantine equation (3.10). In particular, $\operatorname{mult}(X)=\operatorname{mult}(Y)$.

Proof. With notation as in Lemma 3.11 we can write the weights $\left(\lambda_{0}, \lambda_{1}, \lambda_{2}\right)$ of $X$ in the form $\lambda_{i}=c_{i} a_{i}^{2}$, where the $c_{i}$ are square-free positive integers. From Proposition 3.3 we know that $Y$ has weights

$$
\left(\lambda_{1}, \lambda_{2}, \frac{\left(\lambda_{1}+\lambda_{2}\right)^{2}}{\lambda_{0}}\right)=\left(c_{1} a_{1}^{2}, c_{2} a_{2}^{2}, \frac{\left(c_{1} a_{1}^{2}+c_{2} a_{2}^{2}\right)^{2}}{c_{0} a_{0}^{2}}\right) .
$$

The final weight is an integer; in particular, it has square-free part $c_{0}$. Thus the $c_{i}$ are invariant under mutation. Furthermore,

$$
\begin{aligned}
\frac{\left(\lambda_{1}+\lambda_{2}+\frac{\left(\lambda_{1}+\lambda_{2}\right)^{2}}{\lambda_{0}}\right)^{2}}{\lambda_{1} \cdot \lambda_{2} \cdot \frac{\left(\lambda_{1}+\lambda_{2}\right)^{2}}{\lambda_{0}}} & =\frac{\left(\lambda_{0} \lambda_{1}+\lambda_{0} \lambda_{2}+\left(\lambda_{1}+\lambda_{2}\right)^{2}\right)^{2}}{\lambda_{0} \lambda_{1} \lambda_{2}\left(\lambda_{1}+\lambda_{2}\right)^{2}} \\
& =\frac{\left(\lambda_{0}+\lambda_{1}+\lambda_{2}\right)^{2}}{\lambda_{0} \lambda_{1} \lambda_{2}} \\
& =\frac{m^{2}}{r k^{2}}
\end{aligned}
$$

and so the ratio $m / k$ is also preserved by mutation. Hence the weights of $X$ and of $Y$ both generate solutions to the same Diophantine equation (3.10). 
Finally we recall that degree is fixed under mutation, hence $\left(-K_{X}\right)^{2}=\left(-K_{Y}\right)^{2}$. But

$$
\frac{m^{2}}{r k^{2}}=\operatorname{mult}(X)\left(-K_{X}\right)^{2}=\operatorname{mult}(Y)\left(-K_{Y}\right)^{2}
$$

and so $\operatorname{mult}(X)=\operatorname{mult}(Y)$.

By combining Propositions 3.3, 3.9, and 3.12 we obtain Proposition 1.1 ,

Remark 3.13. The weights of a fake weighted projective plane correspond to a solution $\left(a_{0}, a_{1}, a_{2}\right)$ of equation (3.10). A one-step mutation gives a second solution via the transformation:

$$
\left(a_{0}, a_{1}, a_{2}\right) \mapsto\left(\frac{m}{k} \frac{a_{1} a_{2}}{c_{0}}-a_{0}, a_{1}, a_{2}\right) .
$$

Example 3.14. Consider $\mathbb{P}^{2}$. In this case $m / k=3, c_{0}=c_{1}=c_{2}=1$, and $(1,1,1) \in \mathbb{Z}_{>0}^{3}$ is a solution of

$$
3 x_{0} x_{1} x_{2}=x_{0}^{2}+x_{1}^{2}+x_{2}^{2} .
$$

Up to isomorphism, there is a single one-step mutation to $\mathbb{P}(1,1,4)$, giving a solution $(1,1,2) \in$ $\mathbb{Z}_{>0}^{3}$ of equation (3.12). Proceeding in this fashion we obtain a graph of one-step mutations corresponding to solutions of (3.12), which we illustrate to a depth of five mutations:

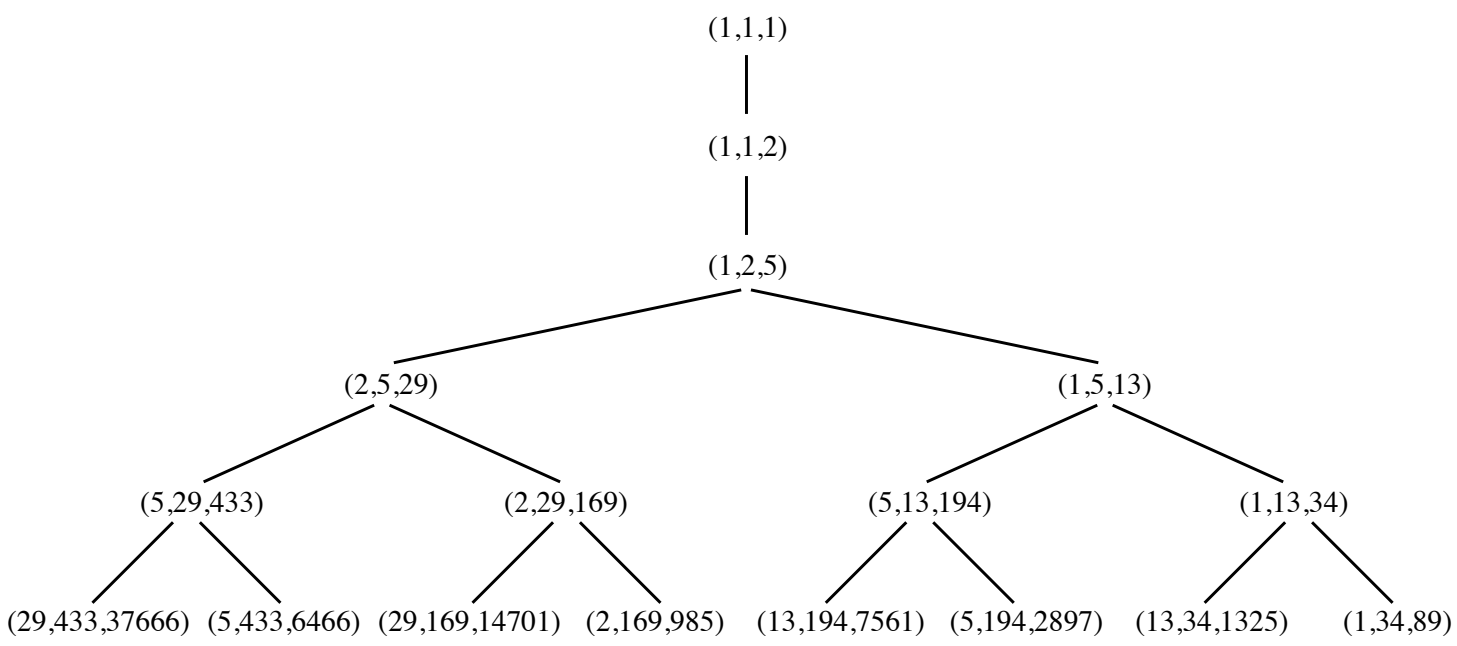

Definition 3.15. The height of the weights $\left(\lambda_{0}, \lambda_{1}, \lambda_{2}\right)$ is given by the sum $h:=\lambda_{0}+\lambda_{1}+\lambda_{2} \in$ $\mathbb{Z}_{>0}$. We call the weights minimal if for any sequence of one-step mutations $\left(\lambda_{0}, \lambda_{1}, \lambda_{2}\right) \mapsto \ldots \mapsto$ $\left(\lambda_{0}^{\prime}, \lambda_{1}^{\prime}, \lambda_{2}^{\prime}\right)$ we have that $h \leqslant h^{\prime}$.

Lemma 3.16. Given weights $\left(\lambda_{0}, \lambda_{1}, \lambda_{2}\right)$ at height $h$ there exists at most one one-step mutation such that $h^{\prime} \leqslant h$. Moreover, if $h^{\prime}=h$ then the weights are the same.

Proof. Without loss of generality suppose we have two one-step mutations

$$
\left(\lambda_{1}, \lambda_{2}, \frac{\left(\lambda_{1}+\lambda_{2}\right)^{2}}{\lambda_{0}}\right) \quad \text { and } \quad\left(\lambda_{0}, \frac{\left(\lambda_{0}+\lambda_{2}\right)^{2}}{\lambda_{1}}, \lambda_{2}\right)
$$


with respective heights $h^{\prime}$ and $h^{\prime \prime}$ such that $h^{\prime} \leqslant h$ and $h^{\prime \prime} \leqslant h$. Since $h^{\prime} \leqslant h$ we obtain $\left(\lambda_{1}+\lambda_{2}\right)^{2} \leqslant \lambda_{0}^{2}$, and so

$$
\lambda_{1}^{2}+\lambda_{2}^{2}<\lambda_{0}^{2}
$$

From $h^{\prime \prime} \leqslant h$ we obtain

$$
\lambda_{0}^{2}+\lambda_{2}^{2}<\lambda_{1}^{2}
$$

Combining equations (3.13) and (3.14) gives a contradiction, hence there exists at most one one-step mutation such that $h^{\prime} \leqslant h$. If we suppose that $h^{\prime}=h$ then

$$
\frac{\left(\lambda_{1}+\lambda_{2}\right)^{2}}{\lambda_{0}}=\lambda_{0}
$$

and equality of the weights is immediate.

The height imposes a natural direction on the graph of all one-step mutations generated by the weight $\left(\lambda_{0}, \lambda_{1}, \lambda_{2}\right)$. Lemma 3.16 tells us that this directed graph is a tree, with a uniquely defined minimal weight.

\section{EXAmple: An infinite number of minimal Weights}

In this section we shall focus on the Diophantine equation

$$
12 x_{0} x_{1} x_{2}=3 x_{0}^{2}+5 x_{1}^{2}+7 x_{2}^{2} \text {. }
$$

Any solution $\left(a_{0}, a_{1}, a_{2}\right)$ such that $\left(3 a_{0}^{2}, 5 a_{1}^{2}, 7 a_{2}^{2}\right)$ is well-formed corresponds to weighted projective space $\mathbb{P}\left(3 a_{0}^{2}, 5 a_{1}^{2}, 7 a_{2}^{2}\right)$ of degree $144 / 105$. One possible such solution is $(2,1,1)$ giving $\mathbb{P}(12,5,7)$. Consider the graph $\mathcal{G}$ of all such solutions. Two solutions lie in the same component if and only if there exists a sequence of one-step mutations between the corresponding weighted projective planes. Furthermore, each component is a tree with unique minimal weight. We shall show that there exists an infinite number of components, and that every component contains at most two solutions; in fact the only component with a single solution is $(2,1,1)$.

4.1. Coprime solutions give well-formed weights. Let $\left(a_{0}, a_{1}, a_{2}\right)$ be a solution of equation (4.1) such that $\operatorname{gcd}\left\{a_{0}, a_{1}, a_{2}\right\}=1$. Clearly this is a necessary condition for the corresponding weights $\left(3 a_{0}^{2}, 5 a_{1}^{2}, 7 a_{2}^{2}\right)$ to be well-formed. We shall show that it is sufficient. For suppose that there exists some prime $p$ such that $p \mid c_{i} a_{i}^{2}$ and $p \mid c_{j} a_{j}^{2}, i \neq j$. Since $p$ cannot simultaneously divide both $c_{i}$ and $c_{j}$, we have that $p$ must divide either $a_{i}$ or $a_{j}$. In particular, $p \mid 12 a_{0} a_{1} a_{2}$ and so, by equation (4.1), $p$ divides the remaining weight $c_{k} a_{k}^{2}$. Similarly, since $p$ can divide at most one of 3,5 , and 7 we see that $p^{2} \mid 12 a_{0} a_{1} a_{2}$ and so $p^{2}$ divides each of the three weights. We conclude that $p \mid \operatorname{gcd}\left\{a_{0}, a_{1}, a_{2}\right\}$, contradicting coprimality. 
4.2. A necessary and sufficient condition for rational solutions when $a_{1}$ and $a_{2}$ are fixed. Fix $a_{1}, a_{2} \in \mathbb{Z}_{>0}$ and consider the quadratic

$$
12 x a_{1} a_{2}=3 x^{2}+5 a_{1}^{2}+7 a_{2}^{2} .
$$

The discriminant is given by

$$
12^{2} a_{1}^{2} a_{2}^{2}-12\left(5 a_{1}^{2}+7 a_{2}^{2}\right)=12\left(5 a_{1}^{2}\left(a_{2}^{2}-1\right)+7 a_{2}^{2}\left(a_{1}^{2}-1\right)\right),
$$

which is always non-negative. The discriminant is zero only in the case $a_{1}=a_{2}=1$, corresponding to the solution $(2,1,1)$ of equation (4.1). Furthermore, we see that a rational solution to equation (4.2) exists if and only if

$$
5 a_{1}^{2}\left(a_{2}^{2}-1\right)+7 a_{2}^{2}\left(a_{1}^{2}-1\right)=3 N^{2}, \quad \text { for some } N \in \mathbb{Z}_{>0} .
$$

4.3. Any rational solution is an integral solution. Suppose that $\alpha, \beta \in \mathbb{R}$ are the two solutions of equation (4.2). We obtain:

$$
\begin{aligned}
\alpha+\beta & =4 a_{1} a_{2}, \\
3 \alpha \beta & =5 a_{1}^{2}+7 a_{2}^{2} .
\end{aligned}
$$

In particular, since the right-hand side in each case is a strictly positive integer, we see that $\alpha, \beta>0$. Furthermore, $\alpha$ is rational if and only if $\beta$ is rational. Since we are only interested in rational solutions, we can assume that both $\alpha$ and $\beta$ are rational. Let us write

$$
\alpha=\frac{n_{1}}{m_{1}} \quad \text { and } \quad \beta=\frac{n_{2}}{m_{2}},
$$

where the fractions are expressed in their reduced form, i.e. $\operatorname{gcd}\left\{n_{i}, m_{i}\right\}=1$. Then

$$
\begin{aligned}
& m_{1} m_{2} \mid 3 n_{1} n_{2}, \\
& m_{1} m_{2} \mid n_{1} m_{2}+n_{2} m_{1} .
\end{aligned}
$$

By (4.7), $m_{2} \mid m_{1}$ and $m_{1} \mid m_{2}$, forcing $m_{1}=m_{2}$. Without loss of generality, from (4.6) we may assume that $m_{1} \mid 3 n_{2}$ and $m_{2} \mid n_{1}$. But then $m_{1} \mid n_{1}$, forcing $m_{1}=m_{2}=1$. Hence $\alpha, \beta \in \mathbb{Z}_{>0}$.

4.4. The values $a_{1}$ and $a_{2}$ are fixed under one-step mutations. We now show that, given a solution $\left(a_{0}, a_{1}, a_{2}\right)$ such that $\operatorname{gcd}\left\{a_{0}, a_{1}, a_{2}\right\}=1$, the values of $a_{1}$ and $a_{2}$ are fixed under one-step mutation. For suppose that

$$
\frac{\left(3 a_{0}^{2}+7 a_{2}^{2}\right)^{2}}{5 a_{1}^{2}} \in \mathbb{Z}
$$

Without loss of generality we may take $\alpha=a_{0}$. We see that $5 \mid 3 a_{0}^{2}+7 a_{2}^{2}=3 \alpha^{2}+3 \alpha \beta-5 a_{1}^{2}$ by (4.5), hence $5 \mid 3 \alpha(\alpha+\beta)=12 a_{0} a_{1} a_{2}$ by (4.4). Since the weights are pairwise coprime, the only possibility is that $5 \mid a_{1}$. Returning to equation (4.8) we see that $5^{2} \mid 3 a_{0}^{2}+7 a_{2}^{2}$, and proceeding as before we find that $5^{2} \mid a_{1}$. Clearly we can repeat this process an arbitrary number of times, increasing the power of 5 at each step. This is a contradiction. The case when

$$
\frac{\left(3 a_{0}^{2}+5 a_{1}^{2}\right)^{2}}{7 a_{2}^{2}} \in \mathbb{Z}
$$


is dealt with similarly.

4.5. An infinite number of components. Set $a_{1}=1$ in condition (4.3). The condition becomes $a_{2}^{2}-1=15 M^{2}$, where $5 M=N$. This is a Pell equation, and Emerson [Eme69] has shown that there exists an infinite number of integer solutions given by a recurrence relation. In this case we see that $a_{2}^{(n)}$ and $M^{(n)}$ are generated by:

$$
\begin{aligned}
a_{2}^{(0)} & =1, & M^{(0)} & =0, \\
a_{2}^{(1)} & =4, & M^{(1)} & =1, \\
a_{2}^{(n+1)} & =8 a_{2}^{(n)}-a_{2}^{(n-1)}, & M^{(n+1)} & =8 M^{(n)}-M^{(n-1)} .
\end{aligned}
$$

Substituting these expressions back into the original quadratic (4.2) gives:

$$
a_{0}^{(n+1)}=2 a_{2}^{(n)} \pm 5 M^{(n)} .
$$

These solutions are coprime (since $a_{1}=1$ ) and so correspond to well-formed weights. We will focus on the smaller of the two solutions, corresponding to the minimum of the two weights. Substituting the expressions for $a_{2}^{(n)}$ and $M^{(n)}$ gives:

$$
\begin{aligned}
a_{0}^{(n+1)} & =2 a_{2}^{(n+1)}-5 M^{(n+1)} \\
& =8\left(2 a_{2}^{(n)}-5 M^{(n)}\right)-\left(2 a_{2}^{(n-1)}-5 M^{(n-1)}\right) \\
& =8 a_{0}^{(n)}-a_{0}^{(n-1)} .
\end{aligned}
$$

Hence we obtain the recurrence relation:

$$
\begin{aligned}
a_{0}^{(0)} & =2, \\
a_{0}^{(1)} & =3, \\
a_{0}^{(n+1)} & =8 a_{0}^{(n)}-a_{0}^{(n-1)} .
\end{aligned}
$$

Remark 4.1. If instead we insist that $a_{2}=1$, we obtain the Pell equation $a_{1}^{2}-1=21 M^{2}$, where $7 M=N$. In this case the recurrence relation is given by:

$$
\begin{aligned}
a_{1}^{(0)} & =1, & M^{(0)} & =0, \\
a_{1}^{(1)} & =55, & M^{(1)} & =12, \\
a_{1}^{(n+1)} & =110 a_{1}^{(n)}-a_{1}^{(n-1)}, & M^{(n+1)} & =110 M^{(n)}-M^{(n-1)} .
\end{aligned}
$$

Proceeding as above we find that

$$
\begin{aligned}
a_{0}^{(0)} & =2, \\
a_{0}^{(1)} & =26, \\
a_{0}^{(n+1)} & =110 a_{0}^{(n)}-a_{0}^{(n-1)} .
\end{aligned}
$$

Hence we have a second infinite family of components of $\mathcal{G}$. Notice that these two families do not exhaust all the possibilities: for example, $a_{1}=5, a_{2}=4$ satisfies condition (4.3), giving the two solutions $(1,5,4)$ and $(79,5,4)$. 
Acknowledgments. Our thanks to Tom Coates, Alessio Corti, and Song Sun for many useful conversations. The authors are supported by EPSRC grant EP/I008128/1.

\section{REFERENCES}

[ACGK12] Mohammad Akhtar, Tom Coates, Sergey Galkin, and Alexander M. Kasprzyk, Minkowski polynomials and mutations, SIGMA Symmetry Integrability Geom. Methods Appl. 8 (2012), 094, pp. 707.

[Buc08] Weronika Buczyńska, Fake weighted projective spaces, arXiv:0805.1211v1, May 2008.

[Con02] Heinke Conrads, Weighted projective spaces and reflexive simplices, Manuscripta Math. 107 (2002), no. 2, 215-227.

[Eme69] Edgar I. Emerson, Recurrent sequences in the equation $D Q^{2}=R^{2}+N$, Fibonacci Quart. 7 (1969), no. 3, 231-242.

[HP10] Paul Hacking and Yuri Prokhorov, Smoothable del Pezzo surfaces with quotient singularities, Compos. Math. 146 (2010), no. 1, 169-192.

[Ilt12] Nathan Owen Ilten, Mutations of Laurent polynomials and flat families with toric fibers, SIGMA Symmetry Integrability Geom. Methods Appl. 8 (2012), 047, pp. 7.

[Kas09] Alexander M. Kasprzyk, Bounds on fake weighted projective space, Kodai Math. J. 32 (2009), 197-208.

[KN12] Alexander M. Kasprzyk and Benjamin Nill, Fano polytopes, Strings, Gauge Fields, and the Geometry Behind - the Legacy of Maximilian Kreuzer (Anton Rebhan, Ludmil Katzarkov, Johanna Knapp, Radoslav Rashkov, and Emanuel Scheidegger, eds.), World Scientific, 2012, pp. 349-364.

[KSB88] J. Kollár and N. I. Shepherd-Barron, Threefolds and deformations of surface singularities, Invent. Math. 91 (1988), no. 2, 299-338.

[Mar80] A. Markoff, Sur les formes quadratiques binaires indéfinies, Math. Ann. 17 (1880), no. 3, 379-399.

[Ros79] Gerhard Rosenberger, Über die diophantische Gleichung $a x^{2}+b y^{2}+c z^{2}=d x y z$, J. Reine Angew. Math. 305 (1979), 122-125.

Department of Mathematics, Imperial College London, London, SW7 2AZ, UK

E-mail address: mohammad.akhtar03@imperial.ac.uk

E-mail address: a.m.kasprzyk@imperial.ac.uk 